\title{
Design of Constant Current Controller for Fast Transient Space Application Protection Scheme Based on Flyback Converter and TPS3700
}

\author{
Poulami Sengupta ${ }^{1}$, Kalpana $\mathbf{P}^{2}$, Prakash $\mathrm{P}^{3}$ \\ ${ }^{1}$ Engr/Sci - SD, URSC/ISRO, Bangalore, Karnataka, India \\ poulami@ursc.gov.in \\ ${ }^{2}$ Section Head URSC/ISRO, Bangalore, Karnataka, India \\ kalpanap@ursc.gov.in \\ ${ }^{3}$ Division Head URSC/ISRO, Bangalore, Karnataka, India \\ pprakash@ursc.gov.in
}

\begin{abstract}
A composite controller fly back topology is proposed for the low voltage, high current (20W) DC - DC converter applications. The controller developed is intended to be used in high performance applications and yet keeping a simple configuration. It combines the feature of Feed-forward and direct sense magnetic coupled feedback control techniques. It also has TPS3700 (window comparator for over and under voltage detection which make the circuit simple and compact, save lot of space. Constant loop gain is achieved in a fly back type DC to DC converter by the use of Feedback to correct for load variation and feed - forward to correct for line voltage variation. Some characteristic provided by these controllers are: rejection of the source variation on the output voltages, better dynamic response, control loop gain doesn't get effected by input voltage and robustness of the design. Furthermore, the operating switching frequency chosen $1 M H Z$ which reduces the overall size and weight of the converter to increase the Power density delivered by the converter. Theoretical analysis and simulations were carried out to analyse the proposed design. The proposed design is verified with a prototype of fly back $d c$ - dc converter fabricated for triple output configuration.
\end{abstract}

Keywords: Fly-back topology, optocoupler-based feedback, TPS3700 IC based window comparator for under and over voltage protection scheme. The operating switching frequency chosen $1 \mathrm{MHZ}$

\section{Salient Features}

- It is a triple output converter, which can be tuned to different specifications.

- Built -in EMI filter meets MIL - STD - 461C

- $1 \mathrm{MHz}$ Switching frequency of operation

- 8 Layer PCB selection make the design more compact and increase overall power density of the converter.

- It has IC basedTPS3700 (window comparator for compact OVP, OCP, OVP and UVP Protection circuits and inrush current protection.

- Tight line regulation is achieved by Feed - forward technique which improves the overall performance of the converter.

- Tight load regulation via direct sensing.

- Efficiency $>80 \%$ forwiderangeof load and line variation.

- Package size: $4.2 * 4$ " *1.6"; weight $=260 \mathrm{gms}$

\section{Introduction}

Compact direct sensed feed - forward controlled $20 \mathrm{~W}$ converter

In case of a conventional 20W DC - DC, Basic topology selected is Fixed ramp voltage controlled forward converter. The major drawback with existing topology:

Lack of production friendliness: There is a need to tune the output voltage and output current over a wide range. This calls for exhaustive tuning of magnetics and compensation elements.

Lack of Optimal design: There is a wide gap between the required output power and rated output power. The maximum capability of output power is underutilized. This leads to low efficiency and specific power

Lack of voltage regulation: All the output is not tightly regulated; indirect regulation approach in all the line is less efficient scheme to a system where both high load and low load can be required parallelly.

Control loop variations cause instability:

In Fixed ramp PWM 20W forward converters operated in Continuous Conduction Mode (CCM) and without any control, the dc output voltage is almost independent of the output current, but it is directly proportional to the dc input voltage. Therefore, it is not easy to achieve a good line regulation in these converters.

Direct sensed Feed - forward technique fly back 20W converter is much advantageous compared to the conventional Miniaturization with better efficiency is the key to opt this technology. Here are the major advantages of Compact direct sensed feed - forward controlled 20Wconverter

A. Advantages of Compact direct sensed feed - forward controlled 20W converter:

When compared to a conventional wire wound, the planar 
magnetic component technology has several advantages.

1) Compact $20 \mathrm{~W}$ Converter with direct sensing will be realized using Fly back topology. It is a triple output converter and direct sensing will ensure tight regulation.

2) The Fly back topology has the advantage of requiring only a single magnetic component. This one, if designed for minimum size, results smaller than the overall volume occupied by both the smoothing inductor plus the power transformer of the equivalent forward converter.

3) Fly back topology is considered the default choice for most power supply requirements due to their somewhat smaller size, lower cost, and comparably high efficiency.

4) It will be realized using Surface mount components and will be realized with a single PCB.

5) Size and Weight of the converter will be reduced to half. Use of surface mount components instead of through hole components will help in achieving this.

6) Realization time will be reduced and it will be made a more production friendly converter.

7) Tight line and load regulation can be achieved through feed forward and feedback technique.

8) Compensation design will be easier as compared to $15 \mathrm{~W}$ fly back circuit in voltage mode control technique.

9) Overall throughput of the converter can be increased.

\section{Study of the Topology}

The Fly back topology is essentially the buck - boost topology with the inductor splits to form a transformer. The transformer not only provides isolation, but by varying the turns ratio, the output voltage can be adjusted. The Flyback is the simplest and most common of the isolated topologies for low - power applications. While they are well suited for high - output voltages, the peak currents are very high, and the topology does not lend itself well to output current above10A.

Fly back topology are most popular choice for cost effective, high efficiency isolated power supply design up to 120 watts they provide circuit isolation, the potential for multiple outputs and the possibility for positive and negative voltages. They can also be regulated over a wide range of input and output voltages condition especially for low load conditions.

\section{Flyback converter}

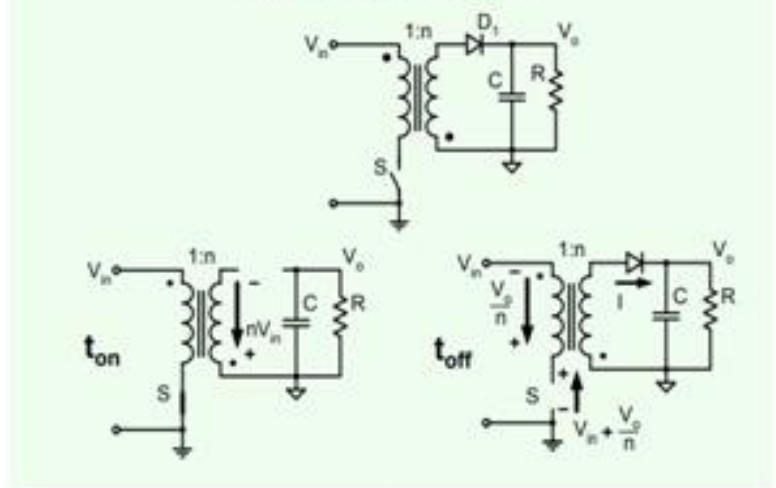
Advantages of Proposed topology:
1) No of components are less
2) Reduction in size and weight
3) Since no of components are less, reliability is more.

\section{B. System Description}

The major blocks of a DC - DC converter are as follows.

1) Power Flow Block - as per converter topology

2) Feedback, Compensation and Control

3) Input filter

4) Start - up circuit

5) Current limit

6) Voltage Protection - input \&output

7) Output voltage sequencing

The aim is to have a topology with the minimum no. of components so as to achieve a maximum specific power for the standard module. Fly back topology is the best option in terms of the minimum no. of components for a DC - DC converter.

\section{Study of the Control Scheme}

There are two modes of control to choose from, viz., voltage mode and current mode. Some of these are outlined below:

\section{Consider the use of current - mode if}

1) The power supply output is to be a current source or very high output voltage.

2) The application is for a DC/DC converter where the input voltage variation is relatively constrained.

3) Modular applications where parallel ability with load sharing is required.

4) In push - pull circuits where transformer flux balancing is important.

Consider voltage - mode (with feed - forward) if:

1) There are wide input line and/or output load variations possible.

2) Particularly with high line - load conditions where the current ramp slope is too shallow for stable PWM operation.

3) High power and/or noisy applications where noise on the current wave form would be difficult to control.

4) Multiple output voltages are needed with relatively good cross - regulation.

5) Applications where the complexities of dual feedback loops.

6) Voltage feed forward control is generally used for better line regulation with voltage mode control.

\section{Proposed controlled scheme: Feed - forward and direct sensed voltage mode}

Some characteristic provided by these controllers are:

- Rejection of the source variation on the output voltages,

- Better dynamic response

- Control loop gain doesn't get effected by input voltage and

- Robustness of the design 
There are three main components in the control loop of a voltage mode DC to DC converter. The three stages are the output stage consisting of the output filter, the modulator gain, and the voltage loop compensation. The two topologies for generating a PWM signal are;

- fixed ramp voltage and

- feed forward voltage topology.

\section{E. Basics of Operation of Voltage feed - forward control:}

The actual PWM signal in a voltage mode regulator is generated by a comparator triggering on a voltage ramp as shown in diagram 1. This ramp is generated from a clock signal and it can be fixed to a particular peak voltage or it can be variable depending on Vin as in the feed forward topology.

When the error amplifier input $\left(\mathrm{V}_{\text {comp }}\right)$ is $0 \mathrm{~V}$ the duty

\section{Vramp $=$ Vin $/ K K=$ constant}

As for buck - boost topology, the input and output relation are

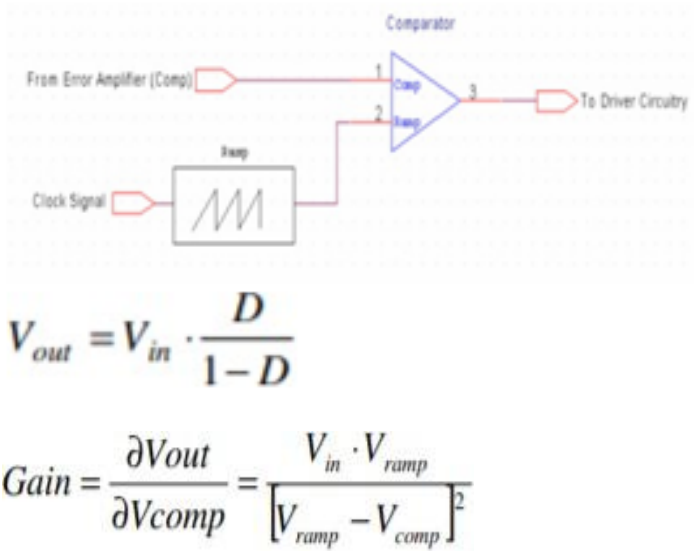

The Modulator gain varies with the input voltage which affects the stability of the converter. The modulator gain and the filter gain are added together to get the total open loop gain for the filter. The important thing to note is that in a fixed ramp topology, as Vin increases so does the total gain of the open loop filter. This in turn pushes out the crossover frequency of the filter which in turn affects the positioning of the poles and zeros of the compensation network. This problem does not exist when the gain of the modulator is fixed as it is in the feed forward topology.

\section{Plan of Implementation}

- Direct sensing of high output line will be done by using voltage divider which fed to opto - coupler which provide isolation between input and output controlled by TL431 shunt regulator.

- TL431 is precise programmable adjustable voltage source. cycle is $0 \%$ meaning the part is off. When the error amplifier voltage $\left(\mathrm{V}_{\text {comp }}\right)$ is equal to peak of the ramp voltage (Vramp) the driver circuitry is at $100 \%$ duty cycle. The duty cycle of the controller is defined as $\mathrm{D}=\mathrm{ton} / \mathrm{T}$ where $\mathrm{T}$ is the Total time defined with respect to the comp voltage and the ramp voltage.

\section{Ton time $=\mathrm{V}$ comp \\ Toff time $=$ Vramp - Vcomp \\ $\mathbf{T}=\mathbf{T}_{\mathrm{on}}+\mathbf{T}_{\mathbf{o f f}}$}

By substituting in the equation:

The gain of the modulator is defined as

In a feed forward topology, the ramp voltage is no longer fixed but varies depending on what the input voltage is.

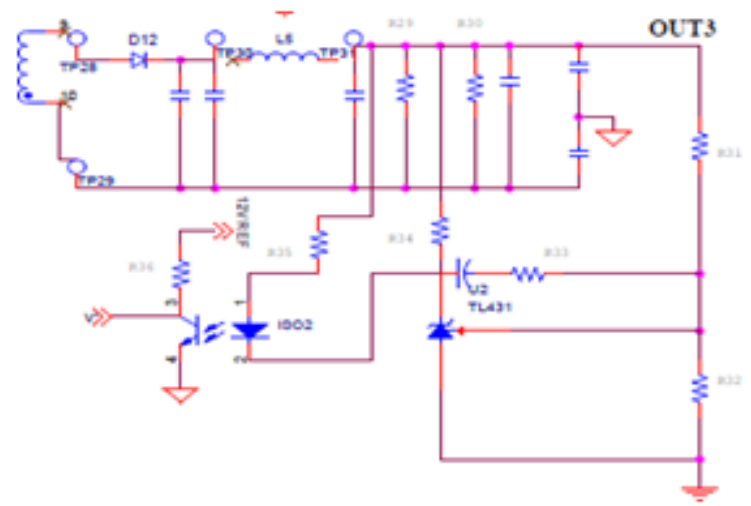

\section{Operation}

- As the voltage increases, the LED of the opto - coupler gets turned on harder, thus the current through the transistor will increase and vfb will decrease.

- If you want the opposite - i. e., vfb increases, then the transistor collector connects to Vdd and the emitter to vfb. Rpullup becomes R pull down.

- Most PWM modulator have negative feedback (Inverted input) so they need to get the feedback from the emitter of the optocoupler.

- Instead of fixed saw tooth ramp, Feed - forward technique is going to be implemented where ramp amplitude will be determined by the input voltage.

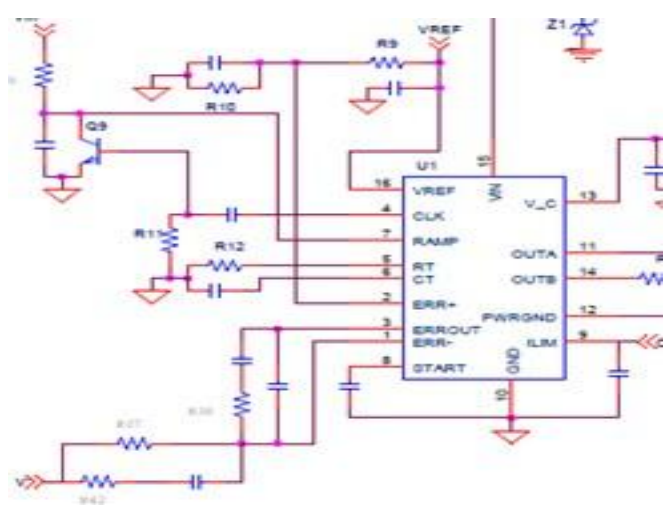




\section{Specifications}

\begin{tabular}{|c|c|}
\hline Parameter & Range \\
\hline Input Voltage & $26-43 \mathrm{~V}, 42-70 \mathrm{~V}$ \\
\hline Outputs & $8 \mathrm{~V} / 2 \mathrm{~A}, 15 \mathrm{~V} / 0.5 \mathrm{~A}$, \\
& $-15 \mathrm{~V} / .5 \mathrm{~A}, 12.5 \mathrm{~V} / 0.05 \mathrm{~A}$ \\
\hline Topology & Flyback Topology \\
\hline Frequency of operation & $500 \mathrm{kHz}$ \\
\hline Efficiency & $80 \%$ \\
\hline Ripple & $50 \mathrm{mV}$ \\
\hline Line regulation & $\pm 1 \%$ \\
\hline Load regulation & $\pm 1 \%$ \\
\hline
\end{tabular}

\section{Design Calculation}

\begin{tabular}{|c|c|}
\hline \multicolumn{2}{|c|}{ Secondary Side Calculations } \\
\hline For Output -1 & 9 \\
\hline For Output -2 & 17 \\
\hline For Output -3 & 17 \\
\hline For HKB & 15 \\
\hline
\end{tabular}

\begin{tabular}{|c|c|}
\hline \multicolumn{2}{|c|}{ Transformer Design } \\
\hline Lpri (uH) & 3.388 \\
\hline Energy (LI^2) & 103.92 \\
\hline Core Selected & $55027-\mathrm{M} 4$ \\
\hline Npri & 9 \\
\hline
\end{tabular}

\begin{tabular}{|c|c|}
\hline \multicolumn{2}{|c|}{ OVP circuit \& UVP circuit calculation } \\
\hline Vfb & 15 \\
\hline V ovp & 16.5 \\
\hline Vref & 2.5 \\
\hline Rtop & 10000 \\
\hline R bottom & 1785.714 \\
\hline
\end{tabular}

\section{Protection Scheme}

TPS3700 window comparator for over and under voltage detection.

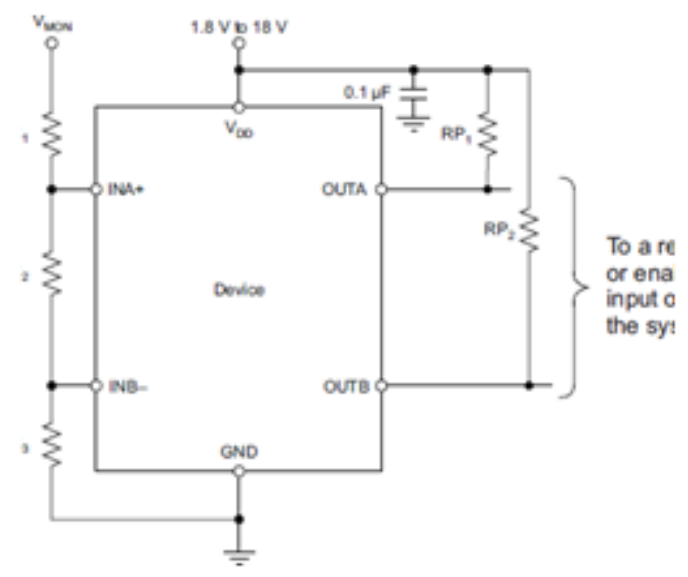

\section{Description}

The TPS3700 wide - supply voltage window operates over a $1.8-\mathrm{V}$ to $18-\mathrm{V}$ range

- The device has two high - accuracy comparators with an internal 400 - $\mathrm{mV}$ reference and two open - drain outputs rated to $18 \mathrm{~V}$ for over - and under voltage detection.

- The TPS3700 can be used as a window comparator or as two independent voltage monitors;

- Low Quiescent Current: 5.5 a(Typical)

- The monitored voltage can be set with the use of external resistors.

\section{Conclusion}

For today's power electronics products, with given quality and reliability, high efficiency, high power density and low cost are getting more and more attention. The power density of converters to be dramatically increased and the power converters to be redesigned. This Compact 20W Converter with direct sensing aims to reduce size and weight of the converter to half. Realization time to be reduced and a more production friendly converter to be realized.

\section{References}

[1] C. Quinn, K. Rinne, T. O'Donnell, M. Duffy and C. O. Mathuna, "A review of planar magnetic techniques and technologies, " APEC2001.

[2] Robert W. Erickson, 'Fundamentals of Power Electronics'2nd Edition, Springer International Edition.

[3] M. A. Saket, N. Shafieiand M. Ordonez, "Planar transformer winding technique for reduced capacitance in LLC power converters, "2016IEEEEnergyConversion Congress and Exposition (ECCE), Milwaukee, WI, 2016, pp.1 - 6.

[4] Ziwei Ouyang, Ole C. Thomsen, Michael A. E. Andersen, Optimal Design and Tradeoff Analysis of Planar Transformer in High - Power DC-DC Converters, IEEE Transactions on Industrial Electronics, vol.59, pp.2800 - 2810 (2012) 